\title{
HARDY-TYPE INEQUALITIES FOR VECTOR FIELDS WITH VANISHING TANGENTIAL COMPONENTS
}

\author{
XINGFEI XIANG AND ZHIBING ZHANG \\ (Communicated by Jeremy Tyson)
}

\begin{abstract}
This note studies Hardy-type inequalities for vector fields with the $L^{1}$ norm of the operator curl. In contrast to the well-known results in the whole space for the divergence-free vector fields, we generalize the Hardy-type inequalities to bounded domains and to non-divergence-free vector fields with tangential components vanishing on the boundary.
\end{abstract}

\section{INTRODUCTION}

This note is devoted to establishing a Hardy-type inequality for vector fields in $L^{1}$ space in 3-dimensional bounded domains. We prove that for a vector field $\mathbf{u}$ with tangential component vanishing on the boundary, the $L^{1}$ norm of $\mathbf{u} /|x|$ can be controlled by the $L^{1}$ norm of $(1+|\ln | x||)$ div $\mathbf{u}$ and the $L^{1}$ norm of curl $\mathbf{u}$.

This work belongs to the field of $L^{1}$ estimates for vector fields. Starting with the pioneering work by Bourgain and Brezis in [2], $L^{1}$ estimates have been well studied by many mathematicians; see $[2-7,10-12,15,16,18]$ and the references therein. In particular, Bourgain and Brezis in [4] obtained the delicate $L^{3 / 2}$ estimate for the divergence-free vectors on the torus $\mathbb{T}^{3}$. Maz'ya in [11] (also see Bousquet and Van Schaftingen's more general result in [6] by introducing the cancellation condition) obtained a Hardy-type inequality for the divergence-free vector field $\mathbf{u}$ (not direct but implied):

$$
\left\|\frac{\mathbf{u}}{|x|}\right\|_{L^{1}\left(\mathbb{R}^{3}\right)} \leq C\|\operatorname{curl} \mathbf{u}\|_{L^{1}\left(\mathbb{R}^{3}\right)} .
$$

This actually gives a partial answer to a problem raised by Bourgain and Brezis in [4, open problem 1]. Bousquet and Mironescu gave an elementary proof of (1.1) in [5].

In this note we consider the problem in bounded domains, in particular with the origin (singularity) being on the boundary. For the case of the origin being in the interior of the domain, one can get Hardy inequalities for vector fields in bounded domains by applying the Hardy inequalities for vector fields in the whole space and by the estimations for vector fields themselves (see the proof of Proposition 2.5). However, if the origin is on the boundary, the usual flattening boundary and the localization by partition of unity do not work. The main reason is that after taking the flattening boundary there would arise the $L^{1}$ norm of $\nabla \mathbf{u}$. This term can't be

Received by the editors March 24, 2014 and, in revised form, September 2, 2014 and October $31,2014$.

2010 Mathematics Subject Classification. Primary 26D10, 42B20, 46 E40.

Key words and phrases. div, curl, Hardy-type inequality, $L^{1}$ data. 
controlled by the $L^{1}$ norm of curl $\mathbf{u}$, when $\mathbf{u}$ is divergence-free, and hence a new approach should be considered.

To get around this difficulty, we apply the Helmholtz-Weyl decomposition for vector fields in bounded domains (see [9, Theorem 2.1]):

$$
\mathbf{u}=\operatorname{curl} \mathbf{w}_{\mathbf{u}}+\nabla p_{\mathbf{u}}+\mathscr{H}_{\mathbf{u}}
$$

where $\mathscr{H}_{\mathbf{u}}$ is the harmonic part depending on the domain and the vector $\mathbf{u}$. Our strategy is to get the estimates for the curl part curl $\mathbf{w}_{\mathbf{u}}$ and for the gradient part $\nabla p_{\mathbf{u}}$ respectively. Thanks to Solonnikov's work in [13, 14] (also see Beirão da Veiga and Berselli's work in [1, p. 606]), the vector $\mathbf{w}_{\mathbf{u}}$ in the curl part satisfies the Petrovsky-type elliptic system, and hence there exists a single Green's matrix $\mathcal{G}(x, y)$ such that

$$
\mathbf{w}_{\mathbf{u}}(x)=\int_{\Omega} \mathcal{G}(x, y) \operatorname{curl} \mathbf{u}(y) d y .
$$

Based on the estimate for the Green's matrix, we can obtain the estimate on the curl part. For the gradient part $\nabla p_{\mathbf{u}}$ we can use the classical elliptic theory to get the estimate.

Before stating the main result, we make the following assumption on the domain:

(O) $\Omega$ is a bounded domain in $\mathbb{R}^{3}$ with smooth boundary; in all cases considered here the class $C^{4}$ will be sufficient. The second Betti number is 0 , which is understood as there are no holes in the domain.

Denote by $\nu(x)$ the unit outer normal vector at $x \in \partial \Omega$. The main result now reads:

Theorem 1.1. Assume that the domain $\Omega$ satisfies the assumption $(\mathrm{O})$. Then for any $\mathbf{u} \in C^{1}\left(\bar{\Omega}, \mathbb{R}^{3}\right)$ with $\nu \times \mathbf{u}=0$ on $\partial \Omega$, we have

$$
\left\|\frac{\mathbf{u}}{|x|}\right\|_{L^{1}(\Omega)} \leq C\left(\|(1+|\ln | x||) \operatorname{div} \mathbf{u}\|_{L^{1}(\Omega)}+\|\operatorname{curl} \mathbf{u}\|_{L^{1}(\Omega)}\right),
$$

where the constant $C$ depends only on the domain $\Omega$.

Remark 1.2. We need to mention that

(i) The reason that there is a log term in Theorem 1.1 is because the divergence operator does not satisfy any cancellation condition. For instance, in the work of Bourgain and Brezis [3] or the work of Lanzani and Stein [10, they also cannot have

$$
\|\mathbf{u}\|_{L^{3 / 2}} \leq C\left(\|\operatorname{div} \mathbf{u}\|_{L^{1}}+\|\operatorname{curl} \mathbf{u}\|_{L^{1}}\right)
$$

on $\mathbb{R}^{3}$ (unless, say, $\operatorname{div} \mathbf{u}=0$ or $\operatorname{div} \mathbf{u}$ is in Hardy space $\mathcal{H}^{1}$ ).

(ii) The method of proof of this theorem in this note is not applicable for vector fields with normal components vanishing on the boundary. We still don't know how to treat the case where the normal components of vector fields vanish on the boundary and the origin (singularity) is on the boundary. For the case of the origin being in the interior of the domain, we can get the same estimate for vector fields with normal components vanishing on the boundary (see Proposition 2.5).

(iii) By a similar discussion, one can get the estimate for elliptic systems associating with Hardy-type inequalities. Let $\mathbf{f} \in C^{1}\left(\bar{\Omega}, \mathbb{R}^{3}\right)$ with $\operatorname{div} \mathbf{f}=0$ in 
$\Omega$ and $\nu \cdot \mathbf{f}=0$ on $\partial \Omega$. If the solution to the elliptic system $\mathscr{L} \mathbf{u}=\mathbf{f}$ can be expressed by

$$
\mathbf{u}=\int_{\Omega} \mathcal{G}(x, y) \mathbf{f}(y) d y
$$

where the Green's matrix $\mathcal{G}(x, y)$ satisfies the inequalities

$$
\text { (i) }|\mathcal{G}(x, y)| \leq \frac{C_{1}}{|x-y|} ; \quad(\text { ii }) \quad\left|\nabla_{y} \mathcal{G}(x, y)\right| \leq \frac{C_{1}}{|x-y|^{2}},
$$

then we have

$$
\left\|\frac{\mathbf{u}}{|x|^{2}}\right\|_{L^{1}(\Omega)} \leq C\left(\Omega, C_{1}\right)\|\mathbf{f}\|_{L^{1}(\Omega)} .
$$

However, this may not be true for the single elliptic equation. The typical example is $\mathscr{L}=\Delta$ with zero boundary condition.

The organization of this paper is as follows. In Section 2, we will give the proof of Theorem 1.1 then we establish a Hardy-type inequality for vector fields with normal components vanishing on the boundary under the condition that the origin (singularity) is in the interior of the domain. In Section 3, Hardy-type inequalities for vector fields with $L^{p}(1<p<3)$ data will be treated, in which the $L^{p}$ norm of $\mathbf{u} /|x|$ can be controlled by the $L^{p}$ norms of $\operatorname{div} \mathbf{u}$ and curl $\mathbf{u}$ whether or not the origin is on the boundary.

Throughout the paper, bold typeface is used to indicate vector quantities; normal typeface will be used for vector components and for scalars.

\section{Hardy-type inequalities For Vector Fields With $L^{1}$ DAtA}

The key step in the proof of the main theorem is the estimate for singular integrals involving the operator curl. This estimate was first obtained by Maz'ya in [1] in the case of the kernel being the Newtonian potential and of the domain being the entire space. The case where the kernel being the Green's function associating with the elliptic operator in the entire space was considered by Bousquet and Van Schaftingen (see [6, Lemma 2.2]). We generalized their kernel to a more general case in bounded domains. The method of our proof goes back to work by Bousquet and Mironescu in [5].

Lemma 2.1. Suppose that the function $A(x, y) \in C^{1}(\bar{\Omega} \times \bar{\Omega})$ for $x \neq y$ satisfies

$$
\text { (i) }|A(x, y)| \leq \frac{C_{0}}{|x-y|^{2}} ; \quad \text { (ii) } \quad\left|\nabla_{y} A(x, y)\right| \leq \frac{C_{0}}{|x-y|^{3}} .
$$

Let $\boldsymbol{\Psi} \in C^{1}\left(\bar{\Omega}, \mathbb{R}^{3}\right)$ with $\operatorname{div} \boldsymbol{\Psi}=0$ in $\Omega$ and $\nu \cdot \boldsymbol{\Psi}=0$ on $\partial \Omega$. Then there exists a constant $C$ depending only on $C_{0}$ such that

$$
\left\|\frac{1}{|x|} \int_{\Omega} A(x, y) \boldsymbol{\Psi}(y) d y\right\|_{L^{1}(\Omega)} \leq C\|\boldsymbol{\Psi}\|_{L^{1}(\Omega)} .
$$

Proof. For simplicity of notation, we let

$$
I_{\Sigma}:=\int_{\Sigma \cap \Omega} A(x, y) \Psi(y) d y .
$$

Then write

$$
\int_{\Omega} A(x, y) \mathbf{\Psi}(y) d y=I_{\{|y|>2|x|\}}+I_{\left\{\frac{|x|}{4}<|y|<2|x|\right\}}+I_{\left\{|y|<\frac{|x|}{4}\right\}} .
$$


The estimate of these integrals is achieved as follows. For all $x, y$ satisfying $2|x|<|y|$, the inequality $|x-y| \geq|y| / 2$ holds. Then using Fubini's theorem, we obtain

$$
\begin{aligned}
\int_{\Omega} \frac{1}{|x|}\left|I_{\{|y|>2|x|\} \mid}\right| d x & \leq \int_{\Omega}|\boldsymbol{\Psi}(y)| \int_{\left\{|x|<\frac{|y|}{2}\right\} \cap \Omega}|A(x, y)| \frac{1}{|x|} d x d y \\
& \leq C \int_{\Omega}|\boldsymbol{\Psi}(y)| \frac{1}{|y|^{2}} \int_{\left\{|x|<\frac{|y|}{2}\right\}} \frac{1}{|x|} d x d y .
\end{aligned}
$$

It is easy to see that the last term of the above inequality can be controlled by the $L^{1}$ norm of $\boldsymbol{\Psi}$. We now estimate the second term in the right side of (2.3) . Using Fubini's theorem again, it follows that

$$
\begin{aligned}
\int_{\Omega} \frac{1}{|x|}\left|I_{\left\{\frac{|x|}{4}<|y|<2|x|\right\} \mid}\right| d x & \leq \int_{\Omega}|\boldsymbol{\Psi}(y)| \int_{\left\{\frac{|y|}{2}<|x|<4|y|\right\}} \frac{1}{|x||x-y|^{2}} d x d y \\
& \leq C \int_{\Omega}|\boldsymbol{\Psi}(y)| \int_{\{|x-y|<5|y|\}} \frac{1}{|y||x-y|^{2}} d x d y .
\end{aligned}
$$

The last term in the above inequality can also be controlled by $\|\Psi\|_{L^{1}(\Omega)}$.

We now estimate the integral involving the term $I_{\{|y|<|x| / 4\}}$. Take the cut-off function $\eta(t)$ such that $\eta(t)=0$ for $t>1 / 2, \eta(t)=1$ for $0<t<1 / 4$, and $\left|\eta^{\prime}(t)\right| \leq 8$. Applying the equality, since $\nu \cdot \boldsymbol{\Psi}=0$ on $\partial \Omega$,

$$
\int_{\Omega} \operatorname{div}\left(y_{i} A(x, y) \eta(|y| /|x|) \Psi(y)\right) d y=0 \quad \text { for } i=1,2,3,
$$

and then using $\operatorname{div} \boldsymbol{\Psi}=0$ in $\Omega$ we have

$$
\begin{aligned}
& \int_{\Omega} A(x, y) \eta(|y| /|x|) \mathbf{\Psi}(y) d y \\
= & -\int_{\Omega} \nabla_{y}(A(x, y) \eta(|y| /|x|)) \cdot \boldsymbol{\Psi}(y)\left(y_{1}, y_{2}, y_{3}\right) d y .
\end{aligned}
$$

The conditions (i) and (ii), for $|y|<|x| / 2$, imply that

$$
\left|\nabla_{y}(A(x, y) \eta(|y| /|x|))\right| \leq C\left(\frac{1}{|x-y|^{3}}+\frac{1}{|x||x-y|^{2}}\right) \leq C \frac{1}{|x|^{3}} .
$$

From (2.6) we can conclude that

$$
\int_{\Omega} \frac{1}{|x|}\left|\int_{\Omega} A(x, y) \eta(|y| /|x|) \boldsymbol{\Psi}(y) d y\right| d x \leq C \int_{\Omega}|\boldsymbol{\Psi}(y)| \int_{\{|x|>2|y|\}} \frac{|y|}{|x|^{4}} d x d y .
$$

The last term in the above inequality can be controlled by $\|\Psi\|_{L^{1}(\Omega)}$. Note that

$$
\begin{aligned}
& \int_{\Omega} A(x, y) \eta(|y| /|x|) \mathbf{\Psi}(y) d y \\
= & I_{\left\{|y|<\frac{|x|}{4}\right\}}+\int_{\left\{\frac{|x|}{4}<|y|<\frac{|x|}{2}\right\} \cap \Omega} A(x, y) \eta(|y| /|x|) \boldsymbol{\Psi}(y) d y .
\end{aligned}
$$

The estimate involving the last term of the above equality can be obtained by a similar proof of (2.5). Thus from (2.7) it follows that

$$
\int_{\Omega} \frac{1}{|x|}\left|I_{\left\{|y|<\frac{|x|}{4}\right\}}\right| d x \leq C\|\Psi\|_{L^{1}(\Omega)} .
$$


Plugging (2.4), (2.5) and the above inequality back into (2.3) we obtain (2.2). We finish our proof.

Remark 2.2. This lemma is still true if we replace the scalar function $A(x, y)$ by a matrix $\mathcal{G}(x, y)$ satisfying the inequalities in (2.1). Indeed, note that we have the inequality

$$
\left\|\frac{1}{|x|} \int_{\Omega} \mathcal{G}(x, y) \boldsymbol{\Psi}(y) d y\right\|_{L^{1}(\Omega)} \leq \sum_{i, j=1}^{3}\left\|\frac{1}{|x|} \int_{\Omega} \mathcal{G}_{i j}(x, y) \Psi(y) d y\right\|_{L^{1}(\Omega)} .
$$

Then by Lemma 2.1,

$$
\left\|\frac{1}{|x|} \int_{\Omega} \mathcal{G}_{i j}(x, y) \Psi(y) d y\right\|_{L^{1}(\Omega)} \leq C\|\boldsymbol{\Psi}\|_{L^{1}(\Omega)} \quad \text { for } i, j=1,2,3 .
$$

Thus, the term in the right side of (2.8) can be controlled by $\|\Psi\|_{L^{1}(\Omega)}$.

By a similar discussion of Lemma 2.1 and applying Fubini's theorem, we can get the estimate of singular integrals for scalar functions.

Lemma 2.3. Suppose there exist the functions $\Psi \in L^{1}(\Omega)$ and $\ln |x| \Psi \in L^{1}(\Omega)$, and assume that the function $A(x, y) \in C^{0}(\bar{\Omega} \times \bar{\Omega})$ for $x \neq y$ satisfies the inequality (i) in (2.1). Then there exists a constant $C$ depending on $C_{0}$ in (2.1) and the domain such that

$$
\left\|\frac{1}{|x|} \int_{\Omega} A(x, y) \Psi(y) d y\right\|_{L^{1}(\Omega)} \leq C\|(1+|\ln | x||) \Psi\|_{L^{1}(\Omega)} .
$$

Proof. Applying the decomposition (2.3), the estimates involving the first and the second terms in the right side of (2.3) can be proved similarly as in the proof of Lemma 2.1. We now estimate the integral involving the term $I_{\{|y|<|x| / 4\}}$. It follows from the inequality $(i)$ in (2.1) that

$$
\begin{aligned}
\int_{\Omega} \frac{1}{|x|} \mid I_{\left\{|y|<\frac{|x|}{4}\right\} \mid d x} & \leq C \int_{\Omega} \frac{1}{|x|} \int_{\left\{|y|<\frac{|x|}{4}\right\} \cap \Omega} \frac{1}{|x-y|^{2}} \Psi(y) d y d x \\
& \leq C \int_{\Omega} \frac{1}{|x|^{3}} \int_{\left\{|y|<\frac{|x|}{4}\right\} \cap \Omega} \Psi(y) d y d x .
\end{aligned}
$$

Then by Fubini's theorem, we have

$$
\begin{aligned}
\int_{\Omega} \frac{1}{|x|}\left|I_{\left\{|y|<\frac{|x|}{4}\right\}}\right| d x & \leq C \int_{\Omega}|\Psi(y)| \int_{\{|x|>4|y|\} \cap \Omega} \frac{1}{|x|^{3}} d x d y \\
& \leq C \int_{\Omega}(1+|\ln | y||)|\Psi(y)| d y .
\end{aligned}
$$

Thus the inequality (2.9) follows.

We now give the proof of the main theorem.

Proof of Theorem 1.1. From the Helmholtz-Weyl decomposition (see 9, Theorem $2.1]$ ), for every $\mathbf{u} \in C^{1}(\bar{\Omega})$ there exists a decomposition

$$
\mathbf{u}=\nabla p_{\mathbf{u}}+\operatorname{curl} \mathbf{w}_{\mathbf{u}},
$$

where the function $p_{\mathbf{u}} \in W^{2, p}(\Omega)$ satisfies (with $1<p<\infty$ )

$$
\Delta p_{\mathbf{u}}=\operatorname{div} \mathbf{u} \quad \text { in } \Omega, \quad p_{\mathbf{u}}=0 \quad \text { on } \partial \Omega,
$$


the vector $\mathbf{w}_{\mathbf{u}} \in X_{n}^{2, p}\left(\Omega, \mathbb{R}^{3}\right)$ with $X_{n}^{2, p}$ being defined by

$$
X_{n}^{2, p}\left(\Omega, \mathbb{R}^{3}\right) \equiv\left\{\mathbf{w} \in W^{2, p}(\Omega): \operatorname{div} \mathbf{w}=0 \text { in } \Omega, \quad \nu \cdot \mathbf{w}=0 \text { on } \partial \Omega\right\}
$$

and the vector $\mathbf{w}_{\mathbf{u}}$ satisfying the elliptic system

$$
\begin{cases}\operatorname{curl} \operatorname{curl} \mathbf{w}_{u}=\operatorname{curl} \mathbf{u} & \text { in } \Omega, \\ \operatorname{div} \mathbf{w}_{u}=0 & \text { in } \Omega, \\ \nu \times \operatorname{curl} \mathbf{w}_{\mathbf{u}}=\nu \times \mathbf{u}=0 & \text { on } \partial \Omega \\ \nu \cdot \mathbf{w}_{\mathbf{u}}=0 & \text { on } \partial \Omega\end{cases}
$$

By the classical elliptic equation theory, we see that the solution $p_{\mathbf{u}}$ of equation (2.11) has the form

$$
p_{\mathbf{u}}=\int_{\Omega} G_{1}(x, y) \operatorname{div} \mathbf{u}(y) d y
$$

and the function $\nabla_{x} G_{1}(x, y)$ satisfies the inequality $(i)$ in (2.1). By Lemma 2.3 we have

$$
\left\|\frac{\nabla p_{\mathbf{u}}}{|x|}\right\|_{L^{1}(\Omega)} \leq C\|(1+|\ln | x||) \operatorname{div} \mathbf{u}\|_{L^{1}(\Omega)} .
$$

We now estimate the term involving the operator curl. Note that the elliptic system (2.12) is of Petrovsky-type (see Solonnikov [13, Theorem 1.1] and [14, Theorem 5.1]; also see the reference [1, p. 606] by Beirão da Veiga and Berselli). Therefore, the solution of (2.12) can be chosen with the representation

$$
\mathbf{w}_{\mathbf{u}}(x)=\int_{\Omega} \mathcal{G}(x, y) \operatorname{curl} \mathbf{u}(y) d y,
$$

where the Green's matrix $\mathcal{G}(x, y)$ is written as

$$
\mathcal{G}(x, y)=\mathbf{G}_{2}(x, y)+\mathbf{R}(x, y) .
$$

The leading term $\mathbf{G}_{2}(x, y)$ satisfies the estimate (see [1, p. 608])

$$
\left|D_{x}^{\alpha} D_{y}^{\beta} \mathbf{G}_{2}(x, y)\right| \leq \frac{C(\alpha, \beta, \Omega)}{|x-y|^{|\alpha|+|\beta|+1}},
$$

and the matrix $\mathbf{R}(x, y)$ satisfies (see [1, p. 610])

$$
\left|D_{x}^{\alpha} D_{y}^{\beta} \mathbf{R}(x, y)\right| \leq \frac{C(\alpha, \beta, \Omega)}{|x-y|^{|\alpha|+|\beta|+1-\gamma}} \quad \text { with } \gamma>0 .
$$

Note that

$$
\operatorname{curl} \mathbf{w}_{\mathbf{u}}(x)=\int_{\Omega} \operatorname{curl}_{x}\left(G^{1}, G^{2}, G^{3}\right)(x, y) \operatorname{curl} \mathbf{u}(y) d y,
$$

where $\left(G^{1}, G^{2}, G^{3}\right)=\mathcal{G}(x, y)^{T}$ is the row vector of matrix $\mathcal{G}(x, y)$, and also we have $\nu \cdot \operatorname{curl} \mathbf{u}=0$ on $\partial \Omega$ since $\nu \times \mathbf{u}=0$ on $\partial \Omega$. Then by the estimates (2.14) and (2.15), Lemma 2.1 is applicable for the vector $\operatorname{curl} \mathbf{w}_{\mathbf{u}}(x)$; thus we get

$$
\left\|\frac{\operatorname{curl} \mathbf{w}_{\mathbf{u}}}{|x|}\right\|_{L^{1}(\Omega)} \leq C\|\operatorname{curl} \mathbf{u}\|_{L^{1}(\Omega)} .
$$

Combining the estimates (2.13) and (2.16), we complete the proof. 
For general domains, we have the following result:

Corollary 2.4. Assume that $\Omega$ is a bounded domain in $\mathbb{R}^{3}$ with smooth boundary. Then for any $\mathbf{u} \in C^{1}\left(\bar{\Omega}, \mathbb{R}^{3}\right)$ with $\nu \times \mathbf{u}=0$ on $\partial \Omega$, there exists a constant $C$ depending only on the domain such that

$$
\left\|\frac{\mathbf{u}}{|x|}\right\|_{L^{1}(\Omega)} \leq C\left(\|(1+|\ln | x||) \operatorname{div} \mathbf{u}\|_{L^{1}(\Omega)}+\|\operatorname{curl} \mathbf{u}\|_{L^{1}(\Omega)}+\|\mathbf{u}\|_{L^{1}(\Omega)}\right) .
$$

Proof. From the decomposition (1.2), we only need to estimate the term $\mathscr{H}_{\mathbf{u}}$. Note that

$$
\mathscr{H}_{\mathbf{u}}=\sum_{i=1}^{m}\left(\mathbf{u}, \mathbf{h}_{i}\right) \mathbf{h}_{i}
$$

where $m$ is the second Betti number of the domain and $\left\{\mathbf{h}_{1}, \mathbf{h}_{2}, \ldots, \mathbf{h}_{m}\right\}$ is the orthonormal basis of $\mathbb{H}_{2}(\Omega)$ (see $[9]$ ):

$$
\mathbb{H}_{2}(\Omega)=\left\{\mathbf{h} \in C^{\infty}\left(\bar{\Omega}, \mathbb{R}^{3}\right): \operatorname{div} \mathbf{h}=0, \operatorname{curl} \mathbf{h}=0 \text { in } \Omega, \nu \times \mathbf{h}=0 \text { on } \partial \Omega\right\} .
$$

Therefore, together with the estimates (2.13) and (2.16), we have

$$
\begin{aligned}
\left\|\frac{\mathbf{u}}{|x|}\right\|_{L^{1}(\Omega)} & \leq C(\Omega)\left(\|(1+|\ln | x||) \operatorname{div} \mathbf{u}\|_{L^{1}(\Omega)}+\|\operatorname{curl} \mathbf{u}\|_{L^{1}(\Omega)}+\sum_{i=1}^{m}\left|\left(\mathbf{u}, \mathbf{h}_{i}\right)\right|\right) \\
& \leq C(\Omega)\left(\|(1+|\ln | x \|) \operatorname{div} \mathbf{u}\|_{L^{1}(\Omega)}+\|\operatorname{curl} \mathbf{u}\|_{L^{1}(\Omega)}+\|\mathbf{u}\|_{L^{1}(\Omega)}\right) .
\end{aligned}
$$

We end our proof.

We give Hardy-type inequalities for vector fields with normal components vanishing on the boundary if the origin (singularity) is in the interior of the domain.

Proposition 2.5. Assume that the domain $\Omega$ is simply-connected with smooth boundary and suppose that the origin $\mathbf{0} \in \Omega$. Then for any $\mathbf{u} \in C^{1}\left(\bar{\Omega}, \mathbb{R}^{3}\right)$ with $\nu \cdot \mathbf{u}=0$ on $\partial \Omega$, we have

$$
\left\|\frac{\mathbf{u}}{|x|}\right\|_{L^{1}(\Omega)} \leq C\left(\|(1+|\ln | x||) \operatorname{div} \mathbf{u}\|_{L^{1}(\Omega)}+\|\operatorname{curl} \mathbf{u}\|_{L^{1}(\Omega)}\right),
$$

where the constant $C$ depends only on the domain $\Omega$.

Proof. Let $d:=\operatorname{dist}(\mathbf{0}, \partial \Omega)>0$. Decomposing the integral and then by Minkowski's inequality, we get

$$
\left\|\frac{\mathbf{u}}{|x|}\right\|_{L^{1}(\Omega)} \leq\left\|\frac{\eta_{1} \mathbf{u}}{|x|}\right\|_{L^{1}(\Omega)}+\left\|\frac{\left(1-\eta_{1}\right) \mathbf{u}}{|x|}\right\|_{L^{1}(\Omega)},
$$

where the cut-off function $\eta_{1}$ is defined by

$$
0 \leq \eta_{1}(x) \leq 1, \quad \eta_{1}(x)=1 \quad \text { in } B_{d / 2}, \quad \operatorname{supp} \eta_{1} \subset B_{d}, \quad\left|D \eta_{1}\right| \leq \frac{4}{d} .
$$

The estimate on the last term of (2.18) is easy to obtain since $\left(1-\eta_{1}\right) \mathbf{u}=0$ in the neighborhood of the origin, and thus by the inequality (3.4) in Remark 3.2 we can conclude that

$$
\left\|\frac{\left(1-\eta_{1}\right) \mathbf{u}}{|x|}\right\|_{L^{1}(\Omega)} \leq C(\Omega)\|\mathbf{u}\|_{L^{1}(\Omega)} \leq C(\Omega)\left(\|\operatorname{div} \mathbf{u}\|_{L^{1}(\Omega)}+\|\operatorname{curl} \mathbf{u}\|_{L^{1}(\Omega)}\right) .
$$


We now estimate the first term in the right side of (2.18). Note that $\eta_{1} \mathbf{u} \in$ $C_{0}^{1}\left(\bar{\Omega}, \mathbb{R}^{3}\right)$. By the fundamental theorem of the representation of vectors (see [17]), we have

$$
\eta_{1} \mathbf{u}=-\frac{1}{4 \pi} \operatorname{grad} \int_{\Omega} \frac{1}{|x-y|} \operatorname{div}\left(\eta_{1} \mathbf{u}\right)(y) d y+\frac{1}{4 \pi} \operatorname{curl} \int_{\Omega} \frac{1}{|x-y|} \operatorname{curl}\left(\eta_{1} \mathbf{u}\right)(y) d y .
$$

Applying Lemma 2.3 for the grad part and using Lemma 2.1 for the curl part, we have

$$
\left\|\frac{\eta_{1} \mathbf{u}}{|x|}\right\|_{L^{1}(\Omega)} \leq C(\Omega)\left(\left\|(1+|\ln | x||) \operatorname{div}\left(\eta_{1} \mathbf{u}\right)\right\|_{L^{1}(\Omega)}+\left\|\operatorname{curl}\left(\eta_{1} \mathbf{u}\right)\right\|_{L^{1}(\Omega)}\right) .
$$

By Hölder's inequality and then applying the inequality (3.4) in Remark 3.2 we have

$$
\|(1+|\ln | x \|) \mathbf{u}\|_{L^{1}(\Omega)} \leq C(\Omega)\|\mathbf{u}\|_{L^{p}(\Omega)} \leq C(\Omega)\left(\|\operatorname{div} \mathbf{u}\|_{L^{1}(\Omega)}+\|\operatorname{curl} \mathbf{u}\|_{L^{1}(\Omega)}\right),
$$

where $1<p<3 / 2$. Combining the inequality (2.21), we have

$$
\left\|\frac{\eta_{1} \mathbf{u}}{|x|}\right\|_{L^{1}(\Omega)} \leq C(\Omega)\left(\|(1+|\ln | x||) \operatorname{div} \mathbf{u}\|_{L^{1}(\Omega)}+\|\operatorname{curl} \mathbf{u}\|_{L^{1}(\Omega)}\right) .
$$

The inequality (2.17) follows immediately by (2.20) and (2.22).

\section{HARDY-Type INEQUALITIES FOR Vector FIELDS WITH $L^{p}$ DATA}

In this section we will deal with Hardy-type inequalities for vector fields with $L^{p}$ data (Theorem 3.3). Before that, we first present the following well-known $W^{1, p}$ estimate for vector fields associating with the $L^{p}$ norms of the operators div and curl.

Lemma 3.1. Assume that $\Omega$ is a bounded domain in $\mathbb{R}^{3}$ with smooth boundary. Let $\mathbf{u} \in W^{1, p}\left(\Omega, \mathbb{R}^{3}\right)$ with $1<p<\infty$. Assume further that

(i) the second Betti number of $\Omega$ is 0 and $\nu \times \mathbf{u}=0$ on $\partial \Omega$ in the sense of trace or

(ii) $\Omega$ is simply-connected and $\nu \cdot \mathbf{u}=0$ on $\partial \Omega$ in the sense of trace.

Then there exists a positive constant $C$ depending only on $p$ and the domain $\Omega$ such that

$$
\|\mathbf{u}\|_{W^{1, p}(\Omega)} \leq C(p, \Omega)\left(\|\operatorname{div} \mathbf{u}\|_{L^{p}(\Omega)}+\|\operatorname{curl} \mathbf{u}\|_{L^{p}(\Omega)}\right) .
$$

Proof. The proof can be found in the literature. Here we give a new proof by using Helmholtz-Weyl decomposition. The estimate on $\|\nabla \mathbf{u}\|_{L^{p}(\Omega)}$ follows from Theorem 3.1 and Theorem 3.2 in [17]. We now estimate $\|\mathbf{u}\|_{L^{p}(\Omega)}$ under the assumption (i). Applying the decomposition (2.10) (also see [9, Theorem 2.1 (3)]) for any vector $\mathbf{v} \in L^{q}(\Omega)$, we have

$$
\int_{\Omega} \mathbf{u} \cdot \mathbf{v} d x=\int_{\Omega} \mathbf{u} \cdot\left(\nabla p_{\mathbf{v}}+\operatorname{curl} \mathbf{w}_{\mathbf{v}}\right) d x=\int_{\Omega}\left(-\operatorname{div} \mathbf{u} \cdot p_{\mathbf{v}}+\operatorname{curl} \mathbf{u} \cdot \mathbf{w}_{\mathbf{v}}\right) d x,
$$

where we have used $p_{\mathbf{v}}=0$ on $\partial \Omega$ and $\nu \times \mathbf{u}=0$ on $\partial \Omega$. Therefore, from Hölder's inequality it follows that

$$
|(\mathbf{u}, \mathbf{v})| \leq\left(\|\operatorname{div} \mathbf{u}\|_{L^{p}(\Omega)}+\|\operatorname{curl} \mathbf{u}\|_{L^{p}(\Omega)}\right)\left(\left\|p_{\mathbf{v}}\right\|_{L^{q}(\Omega)}+\left\|\mathbf{w}_{\mathbf{v}}\right\|_{L^{q}(\Omega)}\right),
$$


where $p$ and $q$ are the conjugate exponents. By the estimate associating with Helmholtz-Weyl decomposition (see [9, Theorem 2.1]), we have

$$
\left\|p_{\mathbf{v}}\right\|_{W^{1, q}(\Omega)}+\left\|\mathbf{w}_{\mathbf{v}}\right\|_{W^{1, q}(\Omega)} \leq C(p, \Omega)\|\mathbf{v}\|_{L^{q}(\Omega)} .
$$

We thus obtain that

$$
|(\mathbf{u}, \mathbf{v})| \leq C(p, \Omega)\left(\|\operatorname{div} \mathbf{u}\|_{L^{p}(\Omega)}+\|\operatorname{curl} \mathbf{u}\|_{L^{p}(\Omega)}\right)\|\mathbf{v}\|_{L^{q}(\Omega)} .
$$

The inequality (3.1) follows immediately. The proof is similar if the case (ii) holds, so we omit it here.

Remark 3.2. In view of the proof of Lemma 3.1, if the index p satisfies $1 \leq p<3 / 2$, then we have

$$
\|\mathbf{u}\|_{L^{p}(\Omega)} \leq C(p, \Omega)\left(\|\operatorname{div} \mathbf{u}\|_{L^{1}(\Omega)}+\|\operatorname{curl} \mathbf{u}\|_{L^{1}(\Omega)}\right) .
$$

Indeed, we replace the inequality (3.2) by

$$
|(\mathbf{u}, \mathbf{v})| \leq\left(\|\operatorname{div} \mathbf{u}\|_{L^{1}(\Omega)}+\|\operatorname{curl} \mathbf{u}\|_{L^{1}(\Omega)}\right)\left(\left\|p_{\mathbf{v}}\right\|_{L^{\infty}(\Omega)}+\left\|\mathbf{w}_{\mathbf{v}}\right\|_{L^{\infty}(\Omega)}\right) .
$$

Then by noting that the space $W^{1, q}(\Omega)$ is continuously imbedded into $L^{\infty}(\Omega)$ for $q>3$, from (3.3) we have

$$
|(\mathbf{u}, \mathbf{v})| \leq C(p, \Omega)\left(\|\operatorname{div} \mathbf{u}\|_{L^{1}(\Omega)}+\|\operatorname{curl} \mathbf{u}\|_{L^{1}(\Omega)}\right)\|\mathbf{v}\|_{L^{q}(\Omega)} .
$$

This gives the inequality (3.4) for $1<p<3 / 2$. For $p=1$, we can obtain (3.4) by Hölder inequality.

We are now in the position to show Hardy-type inequalities for vector fields with $L^{p}$ data.

Theorem 3.3. Assume that $\Omega$ is a bounded domain in $\mathbb{R}^{3}$ with smooth boundary. Let $\mathbf{u} \in W^{1, p}\left(\Omega, \mathbb{R}^{3}\right)$ with $1<p<3$. Assume further that

(i) the second Betti number of $\Omega$ is 0 and $\nu \times \mathbf{u}=0$ on $\partial \Omega$ in the sense of trace or

(ii) $\Omega$ is simply-connected and $\nu \cdot \mathbf{u}=0$ on $\partial \Omega$ in the sense of trace.

Then there exists a positive constant $C$ depending only on $p$ and the domain $\Omega$ such that

$$
\left\|\frac{\mathbf{u}}{|x|}\right\|_{L^{p}(\Omega)} \leq C(p, \Omega)\left(\|\operatorname{div} \mathbf{u}\|_{L^{p}(\Omega)}+\|\operatorname{curl} \mathbf{u}\|_{L^{p}(\Omega)}\right) .
$$

Proof. From the extension theorem in Sobolev spaces ([즈, Theorem 7.25]), we see that for any $\mathbf{u} \in W^{1, p}\left(\Omega, \mathbb{R}^{3}\right)$, there exist a bounded domain $\tilde{\Omega}$ with $\tilde{\Omega} \ll \Omega$ and a vector field $\tilde{\mathbf{u}} \in W_{0}^{1, p}\left(\tilde{\Omega}, \mathbb{R}^{3}\right)$ such that $\tilde{\mathbf{u}}=\mathbf{u}$ in $\Omega$ and

$$
\|\tilde{\mathbf{u}}\|_{W^{1, p}(\tilde{\Omega})} \leq C(p, \Omega, \tilde{\Omega})\|\mathbf{u}\|_{W^{1, p}(\Omega)} .
$$

Note that choosing $\tilde{\Omega}$ depends on $\Omega$. Therefore, the constant $C(p, \Omega, \tilde{\Omega})$ in $(3.6)$ actually depends on the index $p$ and the domain $\Omega$.

By Hardy inequality in $W_{0}^{1, p}(\tilde{\Omega})$ for scalar functions, we have

$$
\left\|\frac{\mathbf{u}}{|x|}\right\|_{L^{p}(\Omega)} \leq\left\|\frac{\tilde{\mathbf{u}}}{|x|}\right\|_{L^{p}(\tilde{\Omega})} \leq C(p)\|\nabla \tilde{\mathbf{u}}\|_{L^{p}(\tilde{\Omega})} .
$$


From (3.6) and (3.7), we immediately get

$$
\left\|\frac{\mathbf{u}}{|x|}\right\|_{L^{p}(\Omega)} \leq C(p, \Omega)\|\mathbf{u}\|_{W^{1, p}(\Omega)} .
$$

We thus obtain (3.5) by Lemma 3.1

\section{ACKNOWLEDGEMENTS}

The authors are grateful to their supervisor, Professor Xingbin Pan, for guidance and constant encouragement. The referees are thanked for many valuable comments on the manuscript that helped to improve the paper. The work of the first author was partly supported by the National Natural Science Foundation of China grants no. 11171111, 11401437 and by China Postdoctoral Science Foundation grant no. $2014 \mathrm{M} 551441$.

\section{REFERENCES}

[1] Hugo Beirão da Veiga and Luigi C. Berselli, Navier-Stokes equations: Green's matrices, vorticity direction, and regularity up to the boundary, J. Differential Equations 246 (2009), no. 2, 597-628, DOI 10.1016/j.jde.2008.02.043. MR2468730(2010h:35291)

[2] Jean Bourgain and Haïm Brezis, On the equation div $Y=f$ and application to control of phases, J. Amer. Math. Soc. 16 (2003), no. 2, 393-426 (electronic), DOI 10.1090/S0894-034702-00411-3. MR.1949165 (2004d:35032)

[3] Jean Bourgain and Haïm Brezis, New estimates for the Laplacian, the div-curl, and related Hodge systems (English, with English and French summaries), C. R. Math. Acad. Sci. Paris 338 (2004), no. 7, 539-543, DOI 10.1016/j.crma.2003.12.031. MR2057026 (2004m:26018)

[4] Jean Bourgain and Haïm Brezis, New estimates for elliptic equations and Hodge type systems, J. Eur. Math. Soc. (JEMS) 9 (2007), no. 2, 277-315, DOI 10.4171/JEMS/80. MR2293957 (2009h:35062)

[5] Pierre Bousquet and Petru Mironescu, An elementary proof of an inequality of Maz'ya involving $L^{1}$ vector fields, Nonlinear elliptic partial differential equations, Contemp. Math., vol. 540, Amer. Math. Soc., Providence, RI, 2011, pp. 59-63, DOI 10.1090/conm/540/10659. MR2807409(2012i:42010)

[6] Jean Van Schaftingen, Limiting Sobolev inequalities for vector fields and canceling linear differential operators, J. Eur. Math. Soc. (JEMS) 15 (2013), no. 3, 877-921, DOI 10.4171/JEMS/380. MR 3085095

[7] Haïm Brezis and Jean Van Schaftingen, Boundary estimates for elliptic systems with $L^{1}$-data, Calc. Var. Partial Differential Equations 30 (2007), no. 3, 369-388, DOI 10.1007/s00526-0070094-9. MR.2332419 (2008m:35063)

[8] D. Gilbarg and N. Trudinger, Elliptic partial differential equations of second order, SpringerVerlag, Berlin and New York, 1998. MR1814364 (2001k:35004)

[9] Hideo Kozono and Taku Yanagisawa, $L^{r}$-variational inequality for vector fields and the Helmholtz-Weyl decomposition in bounded domains, Indiana Univ. Math. J. 58 (2009), no. 4, 1853-1920, DOI 10.1512/iumj.2009.58.3605. MR2542982(2011b:58004)

[10] Loredana Lanzani and Elias M. Stein, A note on div curl inequalities, Math. Res. Lett. 12 (2005), no. 1, 57-61, DOI 10.4310/MRL.2005.v12.n1.a6. MR2122730 (2005m:58001)

[11] Vladimir Maz'ya, Estimates for differential operators of vector analysis involving $L^{1}$-norm, J. Eur. Math. Soc. (JEMS) 12 (2010), no. 1, 221-240, DOI 10.4171/JEMS/195. MR2578609 (2011b:42050)

[12] Irina Mitrea and Marius Mitrea, A remark on the regularity of the div-curl system, Proc. Amer. Math. Soc. 137 (2009), no. 5, 1729-1733, DOI 10.1090/S0002-9939-08-09671-8. MR2470831 (2009j:58002)

[13] V. A. Solonnikov, The Green's matrices for elliptic boundary value problems, I, Trudy Mat. Inst. Steklova 110 (1970), 107-145. MR.0289935(44:7120)

[14] V. A. Solonnikov, The Green's matrices for elliptic boundary value problems. II (Russian), Boundary value problems of mathematical physics, 7, Trudy Mat. Inst. Steklov. 116 (1971), 181-216, 237. MR.0364854 (51 \#1108) 
[15] Jean Van Schaftingen, Estimates for $L^{1}$-vector fields (English, with English and French summaries), C. R. Math. Acad. Sci. Paris 339 (2004), no. 3, 181-186, DOI 10.1016/j.crma.2004.05.013. MR2078071 (2005b:35018)

[16] Jean Van Schaftingen, Limiting fractional and Lorentz space estimates of differential forms, Proc. Amer. Math. Soc. 138 (2010), no. 1, 235-240, DOI 10.1090/S0002-9939-09-10005-9. MR2550188 (2010i:46064)

[17] Wolf von Wahl, Estimating $\nabla u$ by div $u$ and $\operatorname{curl} u$, Math. Methods Appl. Sci. 15 (1992), no. 2, 123-143, DOI 10.1002/mma.1670150206. MR.1149300(93b:26024)

[18] Xingfei Xiang, $L^{3 / 2}$-estimates of vector fields with $L^{1}$ curl in a bounded domain, Calc. Var. Partial Differential Equations 46 (2013), no. 1-2, 55-74, DOI 10.1007/s00526-011-0473-0. MR.3016501

Department of Mathematics, Tongji University, Shanghai 200092, People's Republic OF CHINA

E-mail address: xiangxingfei@126.com

Department of Mathematics, East China Normal University, Shanghai 200241, PeoPle's Republic of ChinA

E-mail address: zhibingzhang29@126.com 